\title{
Knowledge Management for Organisationally Mobile Public Employees
}

\author{
Simon Lambert ${ }^{1}$, Simona Stringa ${ }^{2}$, Gianni Viano ${ }^{2}$, \\ Jacek Kitowski ${ }^{3}$, Renata Słota ${ }^{3}, \mathrm{Krzysztof} \mathrm{Krawczyk}^{3}$, Mariusz Dziewierz ${ }^{3}$, \\ Sabine Delaitre ${ }^{4}$, Miguel Bermejo Oroz ${ }^{4}$, Ana Conde Gómez ${ }^{4}$, \\ Ladislav Hluchy, Zoltan Balogh ${ }^{5}$, Michal Laclavik ${ }^{5}$, \\ $\mathrm{M}^{\mathrm{a}}$ Soledad Fuentes Caparrós ${ }^{6}$, Maria Fassone ${ }^{7}$, and Vito Contursi ${ }^{7}$ \\ ${ }^{1}$ Business \& Information Technology Department, \\ CCLRC Rutherford Appleton Laboratory, Chilton, Didcot, Oxon OX11 0QX, UK \\ S.C.Lamberterl.ac.uk \\ Softeco Sismat SpA, Italy \\ ${ }^{3}$ Cyfronet AGH, Poland \\ ${ }^{4}$ Sadiel SA, Spain \\ ${ }^{5}$ Institute of Informatics, Slovak Academy of Sciences, Slovakia \\ ${ }^{6}$ Consejería de la Presidencia, Junta de Andalucía, Spain \\ ${ }^{7}$ Comune di Genova, Italy
}

\begin{abstract}
Public organisations often face knowledge management problems caused by organisational mobility, the continual and pervasive movement of staff between units and departments. This can be a strength, but also introduces problems of loss of experience and reduced efficiency and effectiveness in working. The Pellucid project is developing a knowledge management system to assist in such situations. The project's three pilot applications are described, and an abstracted view of their knowledge management needs is developed. An outline of key parts of the technical solution is given.
\end{abstract}

\section{Introduction: The Pellucid Project}

Knowledge management has attracted great deal of attention in recent years. It is generally accepted that a large part of the assets of an organisation are knowledge assets [1], and much effort has been devoted to methods, both technological and social, for understanding and augmenting these assets and encouraging their better utilisation[2] is regarded as a seminal work in the field, while many journals in both the business and IT domains (such as Harvard Business Review and IEEE Intelligent Systems) include articles on the theme.

Knowledge management is of as much interest to public administrations as private companies, though has perhaps attracted less attention. Pellucid is a project tackling knowledge management for public bodies under the European Commission's Information Society Technologies (IST) programme. It began in March 2002 and has a duration of 2 years and 8 months. It brings together academic and software developer partners with end-users from across Europe. Its specific motivation comes from the knowledge management issues arising from organisational mobility of employees-th

The original version of this chapter was revised: The copyright line was incorrect. This has been corrected. The Erratum to this chapter is available at DOI: 10.1007/978-3-540-44836-5_33

M.A. Wimmer (Ed.): KMGov 2003, LNAI 2645, pp. 203-212, 2003.

(C) Springer-Verlag Berlin Heidelberg 2003 
e movement of staff from one department or unit to another, a pervasive situation in public organizations.

In this context, knowledge management can be viewed as experience management, since that is what distinguishes the experienced employee from the novice, even if the novice comes from elsewhere in the organisation. The novice might in fact be highly experienced in other parts of the organisation, but lack experience of the new domain of work or the specific procedures that are relevant.

The objectives of Pellucid are to develop a customisable platform for knowledge management, and three pilot applications for validation and demonstration. By a platform is meant a set of methods, tools and reusable components that may be applied to develop further knowledge management applications. The project's approach begins with requirements capture, analysis and generalisation from the three pilot sites within the project, and the development of a general system architecture based on software agents, a paradigm particularly suited to supporting work processes [3]. The system architecture will then be populated, customised to the pilot sites and validated. At the time of writing, the knowledge management needs of the pilot sites have been thoroughly analysed, and the system architecture is undergoing its first implementation. Trials of a first functioning version of the Pellucid system, partially customised to the pilot sites, are planned to take place shortly; it is then expected that valuable feedback will be obtained and refinements will be made to the systems. At that point it will be possible to make a preliminary assessment of the experience of the Pellucid platform in practice.

The rest of this paper explains the nature of organisational mobility in general terms and as it appears in the pilot applications of the Pellucid project. The technical solution is outlined, including a sketch of the Pellucid platform and what will be involved in customisation of it.

\section{Organisational Mobility in Public Organisations}

Organisational mobility is the pervasive movement or circulation of staff from one unit or department within an organisation to another. This is commonplace in public organisations, which may deliberately encourage it as a form of career developmentexposing employees to different working environments and domains of work as a way of building experience. It might also occur due to promotion, and the consequent need to bring in staff from elsewhere in the organisation to fill the newly vacant post.

It is clear that organisational mobility is not necessarily a bad thing: inasmuch as mobile employees bring fresh ideas or experience of other areas, then the organisation can be enriched. Nevertheless, inevitably these employees will find it harder to perform as effectively as more experienced (static) staff, due to their relative lack of specific knowledge. Time must be spent in gaining familiarity, and although there might be training available, and possibly an Intranet as a source of information, these are not sufficient in themselves. It is these problems that Pellucid aims to address.

1 Organisational mobility should be distinguished from geographical mobility, in which individual employees work from different locations, possible widely distributed. In organisational mobility the working location will generally be fixed, but the position will be filled by different individuals over time. 
The Pellucid project includes in organisational mobility the case of high staff turnover. Here the mobile staff are actually coming from outside the organisation, thus without even the knowledge of internal people, but the problems of knowledge management are similar.

\section{The Pellucid Pilot Applications}

\subsection{Requirements Acquisition Methodology}

The determination of the user requirements for the Pellucid platform has followed a previously defined methodology that allows the users to participate in the definition of the system from the very beginning of the project. This methodology is based on three steps.

1. Semi-structured interviews, to get the implicit information / knowledge from the users. This type of interviews is very useful in order to capture the vocabulary, the opinions and the general users requirements.

2. Analyses of relevant documents and users organisations, to get the explicit information/knowledge.

3. Scenarios, to capture end-users' needs in their context and focus on the specific aspects of knowledge management. Scenarios also provide a way to validate the results of the interviews and analyses, with the end users.

The results of these steps allow the identification of the requirements, of the knowledge management improvements and of the different actors involved in the process.

For each pilot application, each scenario has been described by gathering and analysing the information about the activities, the actors and their roles, the people interactions, the information sources and the information flows.

\subsection{Pilot Application 1}

The pilot application of the Comune di Genova (Italy) will address the support to organisational mobility among the several areas of the Traffic and Mobility Management Department of a large city Administration. The services managed range from the strategic traffic planning, to the definition and design of traffic circulation plans within the overall road network, to the simulation activities, to the design, installation and maintenance of traffic signs and signalling systems and to daily operation and management of technical systems and facilities controlling traffic and mobility within the road network.

Particularly, the Pellucid platform will be developed and tested within the process for the installation and maintenance of the traffic light plants. This procedure involves many actors and is constituted by several phases. It is possible to schematize them in the following ones:

1. Input: to point out the need for a new traffic light;

2. Preliminary analysis: to underline particular problems in the interested zone;

3. Verification: to collect information about pedestrian, traffic flows, accdents,..;

4. Design: to define in detail the plant;

5. Definitive evaluation: to verify all the aspects of the project; 
6. Administrative procedure: to define the financial and administrative aspects;

7. Execution: to install the traffic light;

8. Testing: to verify the correct running of the system;

9. Maintenance.

It is not a very complex process because generally there is not overlap during the different phases, but the task is not straightforward because it comprises several aspects, both technical and administrative.

The main actors acting during this process are the Mobility and Transport Directorate of the Comune di Genova, the urban police, the area district, the public transport company, and the company that executes the works. There are not only internal actors to the organisation of the Municipality of Genoa but also external, and therefore the experience and the knowledge obtained by the technical staff during the job are very relevant in order to carry out the task more efficiently. A major source of knowledge management problems is the large number of actors involved, and the need for co-ordination among the several departments in order to get the optimal final result. Less experienced employees have less awareness of who they need to contact at what stage among the external actors, how this contact should be made (by email, by letter, whether there is a standard form for a letter, ...), and how long the actor normally takes to reply. These are all examples of knowledge that an experienced employee will have acquired, and they should be shared effectively.

\subsection{Pilot Application 2}

The Mancomunidad de Municipios del Bajo Guadalquivir (MMBG) is an organisation created by eleven local authorities with the main objective of contributing to the social and economic development of an area with 250,000 inhabitants in the southern Region of Andalusia (Spain). The particular problem of MMBG is the wide range of tasks that must be handled by its employees, from handcraft to IT related activities. This variety of areas in the working environment requires a high degree of flexibility among the employees, and expertise is scarce and very valuable. In this situation, the need for knowledge capitalisation and for reuse of previous experiences is very critical, as it would lead to an increase of the efficiency and would allow for a better use of the human and technical resources.

The pilot application that will be validated at the MMBG concerns the Management of publicly funded Projects among this complex organisation. This will include all the tasks to be performed from the very early stages of the project (definition of the idea, preparation of the proposal and submission to the funding authority) to the justification of the project's costs and activities, and the preservation of all the documents generated during the project execution.

The main problems to be addressed concerning the Management of Projects are due to the lack of integration among the departments involved in the different project phases, and to the non-existence of a solid information structure to provide support for external and internal issues, such as contacting potential providers or preparing a proposal. This is however not simply an IT issue; it reflects the varied and illstructured nature of the work done. Thus staff find themselves without support-not only new employees but also experienced staff who can be involved in very different proposals and projects, so that they could also be considered as 'organisationally mobile employees'. 


\subsection{Pilot Application 3}

The Consejería de la Presidencia integrates and co-ordinates several Regional Bodies in the eight Administrative Departments which compose the Andalusian Region. Due to their wide scope of application, the several processes performed by this Regional Ministry are large and complex, as they are intended to fulfil the needs of over 7 million inhabitants, and have to establish collaborative working with private and public organisations.

Although Pellucid will mainly concentrate on employees at middle and upper levels of the organisations, the so-called 'knowledge workers', the objective of the Pilot application of the Consejería de la Presidencia is to test the viability of Pellucid at low-level organisationally scenarios with common problems related to high staff mobility.

The testing environment for Pellucid among this pilot site will be the Call Center for Management and Resolution of Fixed Telephony Breakdowns of the Telecommunications Corporate Network of the Andalusian Regional Government, and more precisely, the induction of a new agent into this Call Centre.

Call Centre agents have to adapt in a very short time to a very specific working environment, getting a slight 'on-the-job' training support. These employees suffer from a very high mobility degree, and it has been proven that, due to their special working conditions, they use to keep their position 6 months as an average. This very unstable environment makes the need for:

- improving the learning process of new employees, what is very critical to achieve the needed homogenisation of behaviours;

- registering, keeping and exploiting efficiently the experience and knowledge of the agents before they leave the Call Centre or leave their jobs. This is critical to avoid the complete loss of their experience.

There is already available a Workflow Management System supporting the managing and resolution of the breakdowns, the Vantive System, but this system does not cover all the knowledge management requirements. Therefore, additional support will be needed from Pellucid for the complete process life-cycle, in order to guarantee the optimal integration of the new agent in the call centre, and thus the quality of the services provided.

\section{Generalising Knowledge Management Needs}

Although the selected procedures for each pilot application are quite different, all the involved employees have to deal with similar issues when performing their daily activities. Each procedure has to be accomplished by contacting several external and internal actors and by producing and evaluating documents, and all these, meeting the established deadlines.

This leads to a generalised set of knowledge management needs that are of wide applicability in many organisations.

1. Contact management, the capability to get in touch with the 'right person at the right time' when performing activities involving several actors. 
2. Document management, that deals with knowledge about how to prepare documents and how to find and reuse existing documents.

3. Critical timing management, that deals with activity planning to prevent problems or failures due to possible delays by evaluating how critical activities are, which conditions can influence their accomplishment, which symptoms can warn about possible problems arising and are able to prevent problems.

In these three areas, what distinguishes the experienced employee from the newcomer is the knowledge that he/she has available at their fingertips: knowledge about who to contact and how for particular information or to get a job done; knowledge of what documents are available for reference and reuse, and how to create new ones; and knowledge of how long certain tasks are expected to take or certain organisations to reply to queries. Across these three needs, there is a common 'experience management' cycle with three steps.

1. Capture, the observation of employees' behaviour in relation to the work process, for example adding contacts with some comments, choosing one document rather than another.

2. Capitalisation, the creation of new knowledge based on the observation of all employees, making inferences to update (for example) the organisation of contacts for all employees.

3. Return and reuse, the optimised presentation of relevant information according to the activity the employee is carrying out. Another way to return the experience captured is to present a 'roadmap' (of documents/contacts), a plan of future activities with attached the list of contacts needed for each.

This cycle is seen as key to the effective operation of the Pellucid knowledge management solution, because it allows for continuous improvement of behaviour.

\section{An Approach to a Technical Solution}

\subsection{The Architectural Basis}

The Pellucid project is developing a customisable platform to aid with the knowledge management needs identified in the foregoing sections. This platform is based on software agents, arranged in a multi-level structure as shown in Fig. 1.

The three levels each containing a number of agent classes with defined responsibilities.

1. The interaction layer is responsible for managing the interaction with individual users, presenting information in a personalised and timely way and allowing queries and evaluations to be made.

2. The process layer is where the main knowledge management functionality resides, providing assistance on particular activities and roles and interacting with the workflow management system (if one exists).

3. The access layer is responsible for searching and retrieval from document repositories.

In addition there is the Organisational Memory, storing a history of the processes and interactions as a basis for continuous improvement of the knowledge management functionality. 


\section{End users}

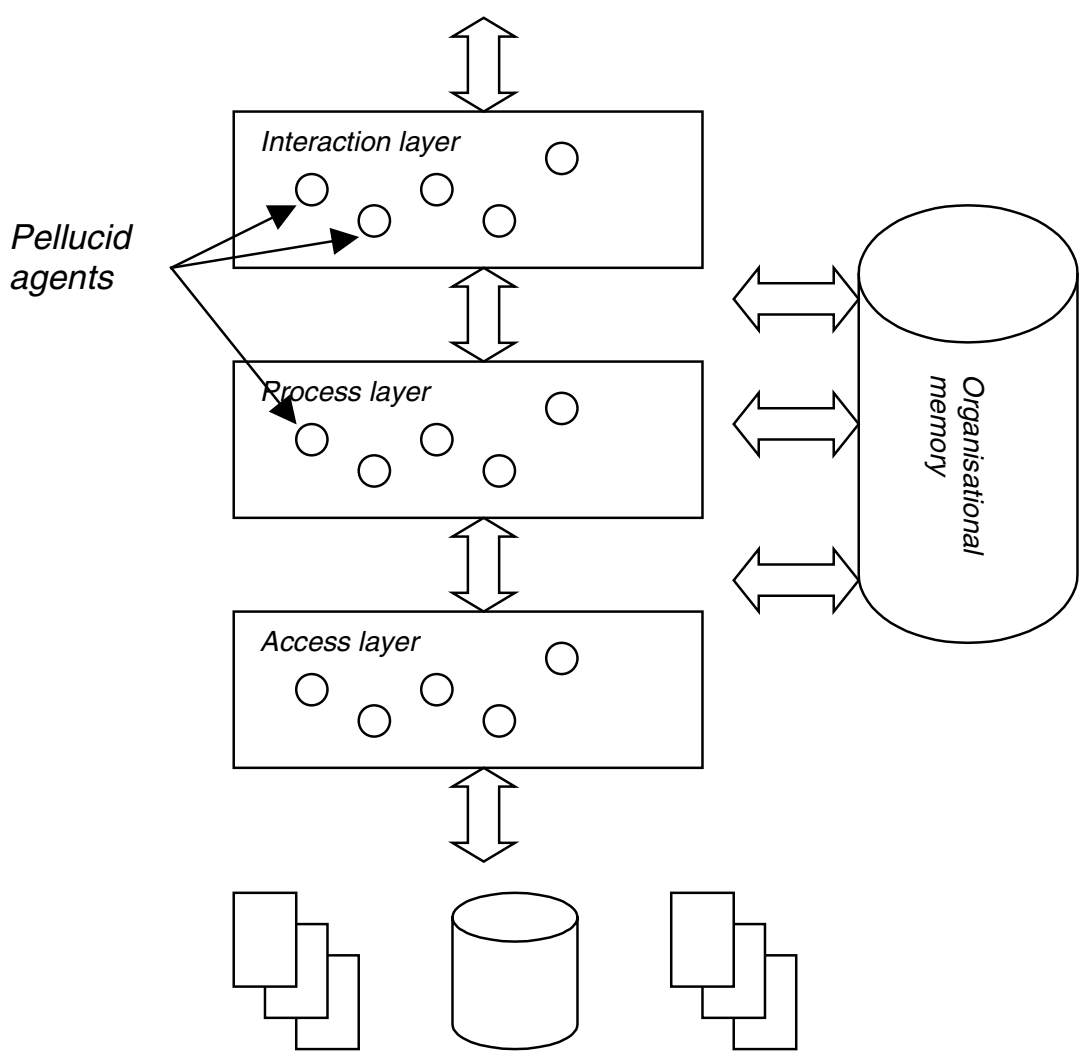

Document repositories

Fig. 1. Block diagram of the Pellucid architecture low.

The three main elements of the Pellucid platform are described in more detail be-

\subsection{The Use of Software Agents}

Agent Oriented Programming (AOP) [4, 5] extends the abilities of Object Oriented Programming (OOP). Classes or objects in OOP are replaced by agents. In OOP an object can call another object by methods and has to know the definitions of the methods of another object. In AOP agents are communicating and acting by message exchange; this is an advantage compared to method calling in OOP which can throw exception. Inter-agent communication is based on content languages and agent ontologies for specific domains of communication. An ontology is a structured representation of the entities and relationships between them in a particular domain, and as 
such forms a common basis on which agents communicate and perform their reasoning.

Pellucid uses so-called Intelligent Agents based on FIPA standards for intelligent knowledge management. Using agents makes Pellucid component-based, more scalable and more customizable [6]. Designing the Pellucid system based on agents helps to focus on individual tasks which are solved by a single agent, together creating a complex system. The use of ontologies makes Pellucid customisable for different problem domains by changing the domain ontology; some parts of the ontology will remain unchanged, for example those relating to general organisational properties and processes.

\subsection{Organisational Memory}

Organisational memories [7] are motivated by the desire to preserve and share the knowledge and experiences that reside in an organisation. As such, they focus on capturing the knowledge, storing it, and making it accessible to analysis and reasoning processes. In the Pellucid context the role of the Organizational Memory (OM) fits tightly the above conceptual definition. It is the central repository of all data and knowledge captured, accumulated and created during the system lifecycle, and provides the raw elements for the agents' reasoning.

The OM contains two main item classes: facts and inferences. The first comprises all kind of data gathered thought the interaction with the users. The second includes the initial knowledge base and the results of reasoning and learning processes taking places in the Pellucid agents on the basis of what is present in the OM. Thus the OM is a living and evolving repository that will support experience capturing (the facts), capitalisation (facts are transformed into inferences) and reuse (from inferences).

The dynamic nature of the OM implies a continuous increase of stored data that are used by agents for knowledge base evolution. The conceptualisation process, together with time flowing, can be at the basis of the progressive and gradual reduction of data relevance thus allowing pruning to maintain the dimension of the OM within a sensible range. The OM permits data mining: uniformities and patterns in workflows and behaviours may be detected and fed back into the active knowledge management components, thereby dynamically enhancing their performance. Knowledge management systems must never be static, or they will become obsolete; such ongoing updating is very necessary.

The OM will not provide knowledge management functions itself, but only storage. Agents are responsible for knowledge management and interact with the OM module, which provides services to support the agents' activity. The OM is mainly composed of three parts.

- An internal database, which is the central storage system and provides the persistence functionality. Object-oriented database technology is under investigation for the effective implementation of this part.

- A communication layer able to interact with agents using FIPA's ACL, a language quite far from usual 'database query languages'. Communication will adopt the Pellucid ontology rather than typical database terminology.

- An internal indexing service to analyse stored data for index preparation and object cluster discovery. The external agents can modify indexing principles to follow the knowledge discovery process. 


\subsection{Document Search and Retrieval}

There is an agent class, the Information Search and Access Agent (ISA), which receives requests for document search and retrieval from other components of the Pellucid system. The environment that the ISA is working in consists of the following elements [8].

Organisational repositories with information assets stored in documents, databases, filesystems, etc. These are needed by the organization to perform its standard activities.

- Document ontologies.

- Document metadata, that describe documents in the organization: (a) businesslevel metadata to express content, context and location of the documents, and (b) technical-level metadata, to express the way to accessing information from a particular type of the repository.

- Full-text search engine index, that optimizes search for documents described in terms of their contents.

Search capabilities are based on smart usage of metadata repositories and full-text search engine. Metadata repositories store descriptions of all documents in the organisation and the way to accessing, while full-text search engine maintains indices vital for providing the user with fast and precise response to his query.

The first method of searching (with metadata) is especially useful when locating documents strongly connected with workflow activities, since with every change of activity there is a need to provide the user with relevant information concerning this current activity. In this case the responsibility is to bring to the user a list of corresponding documents. They are selected by means of request context used for matching knowledge contained in document or domain ontologies and business-level metadata.

To searching and accessing documents, which are not strictly connected to any particular activity, searching with full-text search engines is more adequate. In this case query to ISA is expected to contain some hints concerning documents content specified by means of words or phrases, which should or shouldn't appear in the documents. This kind of request is realized with the help of indices prepared earlier with the document indexing engine. It is up to the agent to make decision about the way it performs the search; it could be a simple search in metadata repository or aggregated search in metadata and in indices repositories from the full-text search engine.

\subsection{Customisable Platform}

The Pellucid platform will consist of elements that are in some way or another reusable. The type of reuse will depend on the nature of the element itself: for example, the software representing an agent class might be stripped down into a template for future application, and/or provided as an example to be modified. Clearly this will depend on the experience gained during the project. The general reusable elements are expected to include the following:

- ontologies (general and domain-specific);

- user interface (Web application); 
- system interfaces (to document repositories and workflow management systems);

- the agents themselves;

- the organisational memory.

The following table indicates a preliminary view of how the elements can be provided in the platform, and the corresponding kinds of customisation that will be applicable to them.

Table 1. Customisable elements in the Pellucid platform

\begin{tabular}{|l|l|}
\hline Element provided in platform & How customised \\
\hline Core (e.g. of ontology) & Expand/Populate/Possibly adapt \\
\hline Template (e.g. of agent class) & Fill in/Populate \\
\hline Prior case (e.g. of agent class) & Adapt \\
\hline $\begin{array}{l}\text { Building blocks (e.g. of organisa- } \\
\text { tional memory) }\end{array}$ & Assemble \\
\hline $\begin{array}{l}\text { Guidelines (e.g. for interfacing to } \\
\text { document repositories) }\end{array}$ & Implement \\
\hline $\begin{array}{l}\text { Specifications (e.g. for interfacing to } \\
\text { workflow management system) }\end{array}$ & Implement \\
\hline
\end{tabular}

Further investigation is needed to establish the best way of including each element in the platform.

\section{References}

1. Strassman, P.A., 'The value of computers, information and knowledge', 1996, available from website http://www.strassmann.com/pubs/cik/cik-value.shtml.

2. Davenport, T.H. \& Prusak, L., Working Knowledge: How Organizations Manage What They Know, Harvard Business School Press, 1998.

3. Jennings, N. et al., 'Autonomous agents for business process management', Int. Journal of Applied Artificial Intelligence 14 (2) 145-189, 2000.

4. Ciancarini, P. \& Wooldridge, M. (eds.), Agent-Oriented Software Engineering, Springer Verlag, 2001.

5. Jennings, N., 'Agent-based Computing: Promise and Perils', Proc. 16th Int. Joint Conf. on Artificial Intelligence, Stockholm, Sweden, 1429-1436.

6. Review of Software Products for Multi-Agent Systems, published by AgentLink, June 2002.

7. Abecker, A., et al., 'Toward a technology for organizational memories', IEEE Intelligent Systems, May/June 1988.

8. Słota, R., Krawczyk, K., Dziewierz, M., Kitowski, J., Lambert, S., 'Agent paradigm for accessing document repositories in Pellucid platform', EuroWEB 2002 Conference, Dec. 1719, 2002, Oxford, UK, accepted. 